\title{
Der Einsatz verschiedener Korpusmethoden und -verfahren zur Qualitäts- und Konsistenzsicherung am Beispiel der Ermittlung und Dokumentation von Synonymen und Antonymen
}

\author{
1. Einleitung \\ 2. Der Einsatz verschiedener korpus- \\ linguistischer Verfahren \\ 2.1 Methodologische Vorüberlegungen \\ 2.2 Chancen und Probleme der korpus- \\ gesteuerten Ermittlung \\ 2.3 Chancen und Probleme der korpus- \\ basierten Ermittlung
}

\author{
3. Methoden konsistenter sinnrelationa- \\ ler Vernetzungsstrukturen \\ 4. Schlussbemerkungen \\ 5. Literatur \\ 5.1 Wörterbücher \\ 5.2 Literatur \\ 5.3 Elektronische Quellen
}

\begin{abstract}
Dieser Beitrag beleuchtet die Vor- und Nachteile korpusgestützter lexikografischer Methoden zur Ermittlung und Dokumentation sinnrelationaler Ausdrücke eines Stichwortes. Konkrete Beispiele aus der Praxis des elexiko-Wörterbuchs dienen der Veranschaulichung von Chancen als auch von konkreten Problemen, die die eingesetzten Methoden mit sich bringen. Für die Gewinnung potentieller Synonyme und Antonyme nutzt elexiko zwei unterschiedliche Verfahren, die mit verschiedenen Prämissen an ein Korpus als Datengrundlage herantreten (cf. Tognini-Bonelli 2001). Das korpusgesteuerte/korpusgeleitete Verfahren der Kollokationsanalyse und die zugrunde liegende Ermittlung von Ausdrücken mit verwandten Kollokationsprofilen (related profiles) (cf. Belica 2011) dienen der empirischen und statistischen Absicherung von sprachlichen Phänomenen. Sie erweisen sich aber als lückenhaft in Bezug auf einige Kontexte, in denen semantisch-konzeptuelle Beziehungen der Ähnlichkeit oder des Gegensatzes realisiert, aber nicht mit Korpustools erfasst werden. Mit der Anwendung der in elexiko komplementär genutzten korpusbasierten Vorgehensweise können diese Lücken teilweise gefüllt werden. Das Zusammenspiel beider Korpusansätze hat sich in der lexikografischen Praxis prinzipiell als vorteilhaft erwiesen, bringt jedoch auch Erkenntnisse zum Vorschein, die bisher weder linguistisch erfasst noch lexikografisch dokumentiert wurden und löst nicht, wie teilweise angenommen, das Problem inhaltlicher Inkonsistenzen (cf. Paradis/Willners 2007). Diese Aspekte werden anhand von konkreten Korpusbeispielen und Wörterbucheinträgen illustriert.
\end{abstract}

Als Online-Wörterbuch profitiert elexiko von seinen schnellen Navigationsmöglichkeiten über Verlinkungen. Diese werden auch für sinnrelationale Partnerwörter wie Synonyme und Antonyme angelegt, um diverse Vernetzungsstrukturen nachvollziehbar zu machen. Die Arbeit mit einem Korpus kann bis zu einem gewissen Grad die Konsistenz der bidirektionalen Vernetzungen gewährleisten, sie aber nicht vollständig absichern. In diesem Beitrag wird auch die Frage beantwortet, inwieweit die erwähnten Korpusmethoden dazu beitragen, das gegenseitige Dokumentieren zwischen Synonym- oder Antonympaaren sicherzustellen. Anhand des für diese Zwecke entwickelten Tools vernetziko, einem Vernetzungsmanager, wird gezeigt, warum die Unterstützung zusätzlicher Software für eine konsistente Verlinkung zwischen paradigmatisch miteinander verbundenen Stichwörtern unerlässlich ist (Storjohann/Meyer 2012). 
1. Einleitung

Sinn- und sachverwandte Ausdrücke wie Synonyme und Antonyme ${ }^{1}$ können mit umfangreichen Korpora und den entsprechenden Analysewerkzeugen sehr systematisch und zielgerichtet extrahiert, ggf. linguistisch untersucht und daraufhin lexikografisch beschrieben werden. Dennoch beschreibt kaum ein korpusgestützt erarbeitetes Wörterbuch, das derartige lexikalische Beziehungen zwischen zwei Ausdrücken dokumentiert, seine zugrunde liegenden linguistischen Kriterien, die gewählten korpusmethodologischen Verfahren sowie die dafür eingesetzten Werkzeuge zur Ermittlung von Synonymen bzw. Antonymen. Nur über eine genauere Analyse der Wörterbucheinträge selbst lässt sich feststellen, ob und wenn ja, welche Prinzipien für die Aufnahme von sinnrelationalen Ausdrücken vorliegen. Die Erwartungen an korpusfundierte Angaben schließen neben einer objektiveren Beschreibung des tatsächlichen Sprachgebrauchs vor allem auch ein konsistentes Dokumentieren von Beziehungen für die beteiligten Partnerwörter ein (cf. Paradis/Willners 2007). Die lexikografische Arbeit mit Korpora lässt nicht nur vermuten, dass relevantes Material empirisch, systematisch und auch schneller erfasst werden kann, sondern auch, dass die zugrunde liegende Bidirektionalität einer synonymen oder antonymen Beziehung sich in der konsistenten gegenseitig rückverweisenden Dokumentation zwischen den einzelnen Stichwörtern niederschlagen sollte, vorausgesetzt, sie gehören jeweils zum Stichwortbestand des Nachschlagewerkes.

Ziel einer Untersuchung von Paradis/Willners (2006, 2007) war es, im ersten korpusgestützten Nachschlagewerk des Englischen, dem Collins COBUILD Advanced Learner's English Dictionary (2003), herauszufinden, ob das Vorhandensein oder das Fehlen eines Antonyms bestimmten linguistischen Kriterien unterliegt und wie konsequent Rückverweise in den entsprechenden Einträgen angelegt wurden. Die Annahmen der Autorinnen waren, dass die Arbeit mit Korpusdaten solche Auswahlprinzipien nachvollziehbar macht, weil die Analyse des Sprachmaterials stärker an statistische Auswertungen und Parameter wie die Signifikanz gekoppelt ist. Das Fazit dieser systematischen Studie ergab, dass nur 37\% aller Antonyme konsequent in beide Richtungen verzeichnet sind und dass die Prinzipien zur Aufnahme von semantischen Gegensatzpaaren nicht transparent sind.

We fail to see a clear pattern in the choice of antonymy and the symmetry of presentation [...]. (Paradis/Willners 2006, 216)

Die Mängel wurden besonders bei solchen Paaren deutlich, die den so genannten kanonischen, also äußerst prototypischen Paaren (cf. Jones et al. 2012) wie beispielsweise billig $\rightarrow$ teuer (cheap $\rightarrow$ expensive), schwer/schwierig $\rightarrow$ leicht (difficult $\rightarrow$ easy), alt $\rightarrow$ neu $($ old $\rightarrow$ new), Hass $\rightarrow$ Liebe (hate $\rightarrow$ love), traurig $\rightarrow$ glücklich ( sad $\rightarrow$ happy), ungerade $\rightarrow$ gerade (odd $\rightarrow$ even) zugeschrieben werden, bei denen die Beziehung jeweils nur in einer Richtung angegeben wurde.

1 Für Synonymie wird nachfolgend ein allgemeinsprachliches Verständnis der Bedeutungsäquivalenz zugrunde gelegt. Unter Antonymie werden nicht nur semantisch oder syntaktisch graduierbare Adjektive (cf. Cruse 1986) verstanden, sondern sämtliche Arten des konträren oder kontradiktorischen Beziehungsgegensatzes subsumiert. 
In our opinion as non-native speakers of English, all these pairs deserve to be reversed, e.g. big should be listed as an antonym of small and cheap of expensive. Again, it is not clear to us why these pairings should be helpful for the learner in the above directions only. (Paradis/Willners 2006, 217)

Paradis/Willners (2006, 2007) interpretieren ihren Befund als methodologisches Defizit hinsichtlich der genutzten Verfahren und sie vermuten, dass diese Einträge primär durch korpusbasierte Arbeit zustande gekommen sind. Im tatsächlichen Sprachgebrauch tauchen Antonyme, insbesondere antonyme Adjektive, regelhaft in unmittelbarer kontextueller Umgebung auf (cf. Justeson/Katz 1991, Jones 2002). Lexikografisch sollte man sich daher stärker einer Herangehensweise verpflichten, die die Ermittlung von Kookkurrenzen bzw. statistisch signifikanten sprachlichen Konstruktionen mit einem bestimmten Suchwort zum lexikografischen Prinzip erklärt. Paradis/Willners (2006) schlugen vor, aufgrund des kontextuellen Verhaltens von Oppositionspaaren bei der Ermittlung solcher Beziehungen stärker korpusgesteuert/korpusgeleitet statt korpusbasiert vorzugehen. Sie nehmen an, dass somit stringente Kriterien und eine systematische Vorgehensweise bei der Analyse binärer Beziehungen herangezogen werden können.

Nachfolgend soll anhand des deutschen Nachschlagewerkes elexiko, einem Online-Wörterbuch zur deutschen Gegenwartssprache, gezeigt werden, welche Ergebnisse zu sinnverwandten Ausdrücken aus Korpusdaten erzielt werden, wenn sowohl korpusbasierte als auch korpusgesteuerte Methoden zum Einsatz kommen. Der Beitrag beleuchtet die Vor- und Nachteile beider lexikografischer Ansätze anhand konkreter Beispiele aus der Wörterbuchpraxis und weist auf Chancen und konkrete Probleme hin, die die eingesetzten Herangehensweisen mit sich bringen. Besonderes Augenmerk liegt auf der Beantwortung der Fragestellung, ob der Einsatz korpusgesteuerter Verfahren die von Paradis/Willners (2006) aufgeführten Probleme der Inkonsistenz bidirektionaler Beziehungen löst und die Mängel bezüglich der symmetrischen Aufnahme gegenseitiger Partnerwörter beseitigt. Es soll gezeigt werden, in wie weit damit ein Instrument zur Qualitätssicherung und zur konsistenten Dokumentation von semantisch konträren bzw. äquivalenten Wortpaaren vorliegt.

\section{Der Einsatz verschiedener korpuslinguistischer Verfahren}

\subsection{Methodologische Vorüberlegungen}

Der korpusgesteuerte und der korpusbasierte Ansatz werden im engen korpuslinguistischen Sinn als zwei unterschiedliche, und nicht selten auch gegensätzliche, Herangehensweisen an Korpusdaten verstanden (cf. Tognini-Bonelli 2001). Die Gegensätzlichkeit beruht auf der unterschiedlichen Zielsetzung der jeweiligen Ansätze. Bei der korpusbasierten Methode werden Korpusdaten als Beweismaterial für bestehende Annahmen genutzt. Das Korpus fungiert also als Prüf-, Quantifizierungs- und Validierungsinstrumentarium. Bestehende linguistische Kategorien oder Theorien werden nicht hinterfragt und wie Tognini-Bonelli (2001, 81) hervorhebt ,the commitment to the data as a whole is not ultimately very strict or systematic". Bei der korpusgesteuerten Methode tritt man dagegen ohne Vorannahmen an Korpusdaten heran und wertet diese empirisch mithilfe statistischer Verfahren aus (z.B. mit 
einer Kookkurrenzanalyse), um regelhafte Strukturen zu ermitteln, auf deren Grundlage man zu Beschreibungen oder linguistischen Generalisierungen gelangt.

Während oftmals zwischen beiden Verfahren ein grundlegender Widerspruch gesehen wird (cf. Sinclair 2002), wurde in der lexikografischen Praxis des Projektes elexiko die Erfahrung gemacht, dass sich die beiden Methoden für verschiedene Untersuchungszwecke unterschiedlich eignen. Die explorative automatische Kontextanalyse sichert für frequente Stichwörter objektivere Auswertungen zu kontextuellen Verwendungen und unmittelbaren Partnerwörtern. Für die Ermittlung sinnrelationaler Partner eines zu beschreibenden Ausdrucks hat sich aber gezeigt, dass beim Einsatz beider Korpusverfahren die bestmöglichen Ergebnisse erzielt werden. Für die Gewinnung von Synonymen und auch für die Beschreibung sinnverwandter Ausdrücker verbaler Stichwörter ist eine strikte Trennung der durchaus komplementären Ansätze unvorteilhaft.

\subsection{Chancen und Probleme der korpusgesteuerten Ermittlung}

Bei der korpusgesteuerten Methode geht man davon aus, dass eine bestehende Synonymieoder auch Antonymierelation, so sie regelhaft im Sprachgebrauch vorkommt, als sprachliches Konstrukt emergent aus dem Korpus hervortritt und dass entsprechende Analysewerkzeuge diese auf der Basis statistischer Verfahren aufdecken, ohne dass der Lexikograf/die Lexikografin gezielt nach einer bestimmten Beziehung zwischen zwei Ausdrücken sucht. Auch elexiko nutzt die Ermittlung von Kollokationen/Kookkurrenzen ${ }^{2}$ und anderen statistisch signifikanten Konstruktionen, um bei der semantischen Analyse eines Ausdrucks u.a. auf bedeutungsähnliche bzw. -gegensätzliche Ausdrücke zu stoßen. Dies geschieht mit Hilfe des Korpusanalysemoduls „Statistische Kollokationsanalyse und Clustering“ (Belica 1995). Die Ergebnisse der korpusgesteuerten Untersuchung zeigen zum Teil große Diskrepanzen zu Synonym- bzw. Antonymangaben in Wörterbüchern, die nicht auf Korpusbasis erarbeitet wurden (cf. Storjohann 2006).

Besonders zielführend ist das korpusgesteuerte Verfahren bei der Suche nach adjektivischen Gegensatzwörtern. Es besteht bei Gegensatzpaaren im Allgemeinen die Tendenz kontextuell eng in bestimmten sprachlichen Konstruktionen miteinander aufzutauchen (Justeson/ Katz 1991). Korpusorientierte Studien zeigen, dass die sprachlichen Konstruktionen von Antonymierelationen bestimmte semantische, text- bzw. diskursbezogene Funktionen ausüben (cf. Jones 2002). Die mit Abstand häufigste sprachliche Realisierung stellt dabei die koordinierende Antonymie dar, beispielsweise kleine und große Kinder, schöne oder hässliche Gebäude, sowohl öffentliche als auch private Investoren, entweder feste oder freie Mitarbeiter etc. ${ }^{3}$ Auch Konstruktionen mit negierender Funktion wie etwa, nicht mit legalen, sondern illegalen Mitteln oder nicht dauerhaft, sondern temporär einrichten und mit vergleichender Funktion wie eher dick als dünn oder mehr tot als lebendig zeigen, dass sprachlich die beteiligten Ausdrücke in enger semantischer Umgebung miteinander auftauchen. Das gleiche gilt für Muster, die sich in semantischer Hinsicht auf einen Unterschied

2 Die Termini „Kollokation“ und „Kookkurrenz“ werden in diesem Beitrag synonymisch verwendet und lehnen sich an das korpuslinguistische Verständnis im sinclairschen Sinne an (cf. Sinclair 1991).

3 Cf. auch Jones' (2002) Untersuchung zu ,coordinated antonymy “ und ,ancillary antonymy`. 
beziehen, z.B. zwischen arm und reich, ältere Frauen im Gegensatz zu jüngeren Frauen oder Syntagmen, die einen Übergang markieren, wie der Optimismus schlägt bald in Pessimismus um. Aufgrund des miteinander Kookkurrierens lassen sich derartige Strukturen gezielt aus dem Korpus extrahieren, ohne Vorannahmen darüber zu haben, welche potentiellen Gegensatzwörter kontextuell mit einem Ausdruck $X$ sprachlich realisiert werden. Darüber hinaus gewährleistet ein enges kotextuelles Miteinandervorkommen und die statische Auswertung die Aufdeckung des Partnerwortes $Y$ für das Suchwort $X$ und umgekehrt.

Besonders effektiv ist eine Suche bei monosemen bis gering polysemen Ausdrücken wie etwa privat. Eine korpusgeleitete Suche zweier solcher Muster ergab im elexiko-Korpus zahlreiche, z.T. auch unerwartete Ergebnisse (siehe Abbildungen 1 und 2). An das Korpusrecherche und -analysewerkzeug COSMAS II wurden dafür z.B. folgende Suchanfragen gestellt ${ }^{4}$ :

1. \&privat / Abstand (2w Max) sondern

2. \&privat / Abstand (2w Max) 'UND'.

Die beiden Suchanfragen (Abbildung 1 und 2) zeigen Überlappungen, aber auch Unterschiede in ihren Ergebnissen. Die Wahl der Suchanfragen hat maßgeblichen Einfluss auf ermittelte Ergebnislisten und damit auch auf die mögliche lexikografische Auswahl. Es gilt also, sich der Möglichkeiten typischer Antonymkonstruktionen bewusst zu werden und diese gezielt für die datengeleitete Ermittlung von Gegensatzpaaren einzusetzen.

\begin{tabular}{|lll|}
\hline LLR & Kookkurrenzen & syntagmatische Muster \\
399 & beruflich & $61 \%$ nicht nur beruflich $[\ldots]$ sondern auch privat \\
277 & öffentliche & $62 \%$ nicht nur öffentliche $[\ldots]$ sondern auch private \\
115 & staatliche & $63 \%$ nicht keine staatliche $[\ldots]$ sondern auchleine private \\
86 & dienstlich & $90 \%$ nicht nur dienstlich $[\ldots]$ sondern auch privat \\
45 & gewerblichen & $83 \%$ allerdings keine gewerblichen $[\ldots .$.$] sondern nur private$ \\
33 & geschäftliche & $66 \%$ geschäftliche Termine haben sondern \\
22 & gesellschaftliches & $100 \%$ kein privates sondern ein gesellschaftliches \\
20 & öffentlich-rechtliche & $33 \%$ sondern ... öffentlich-rechtliche \\
17 & landeseigene & $50 \%$ private ... landeseigene \\
16 & politisch & $100 \%$ privat ... politisch \\
12 & offizieller & $75 \%$ offizieller ... sondern \\
11 & berufliches & $100 \%$ sondern ... berufliches \\
9 & städtischer & $50 \%$ privater ... städtischer \\
\hline
\end{tabular}

Abbildung 1: Gegensatzwörter zu privat in Konstruktion nicht nur X, sondern auch Y

4 Die Korpusrecherche beschränkt sich auf die Textsuche, in denen das Lemma privat mit all seinen Wortformen zusammen mit den Ausdrücken sondern und und innerhalb eines Wortabstands von maximal zwei Wörtern auftritt. Darüber hinaus gibt es zahlreiche weitere typische Muster, die Antonympaare syntagmatisch konstruieren (cf. Jones 2002). Für generelle Beispiel-Suchanfragen in COSMAS II siehe auch unter http://www.ids-mannheim.de/cosmas2/web-app/hilfe/seite/suchanfrage/beispiele.html. 


\begin{tabular}{|lll|}
\hline LLR & Kookkurrenzen & syntagmatische Muster \\
11724 & öffentlichen & $58 \%$ öffentlichen $[\ldots]$ und privaten ... \\
5475 & beruflichen & $58 \%$ aus beruflichen $[\ldots]$ und privaten Gründen \\
2740 & öffentlich-rechtlichen & $59 \%$ öffentlich-rechtlichen $[\ldots]$ und privaten ... \\
1963 & gewerblichen & $67 \%$ privaten [und] gewerblichen \\
1814 & staatlichen & $63 \%$ staatlichen $[\ldots]$ und privaten ... \\
1510 & betriebliche & $48 \%$ die betriebliche $[\ldots]$ und private ... \\
1459 & institutionelle Anleger & $53 \%$ für private und institutionelle Anleger \\
1063 & gesetzlichen & $65 \%$ zwischenlder gesetzlichen $[\ldots]$ und privaten Kassenl \\
& & Krankenkassen \\
951 & geschäftliche & $61 \%$ für private und geschäftliche \\
903 & dienstliche & $49 \%$ dienstliche $[\ldots]$ und private ... \\
513 & kommunale & $50 \%$ kommunale $[\ldots]$ und private ... \\
300 & genossenschaftlichen & $80 \%$ derldie privaten [und] genossenschaftlichen Banken ... \\
316 & Sicherheitsdiensten & $71 \%$ von Polizei und privaten Sicherheitsdiensten \\
238 & institutioneller & $61 \%$ privater und institutioneller Kunden ... \\
167 & freigemeinnützigen & $50 \%$ freigemeinnützigen $[\ldots]$ und privaten ... \\
\hline
\end{tabular}

Abbildung 2: Gegensatzwörter zu privat in Konstruktion X und $\mathrm{Y}$

Die großen Vorteile dieser Herangehensweise liegen in folgenden Aspekten. Das Datenmaterial wird explorativ ohne Vorannahmen untersucht, und es werden mithilfe bestimmter Suchanfragen von typischen Gegensatzkonstruktionen investigativ und systematisch alle entsprechenden sprachlichen Realisierungen herausgefiltert. Die linguistischen Regularitäten werden von Analysewerkzeugen aufgedeckt, die Ergebnisse werden nach Signifikanz geordnet und so einer möglichen nachfolgenden lexikografischen Auswertung vorsystematisiert zur Verfügung gestellt. Die Erfahrung, die im Umgang mit solchen Abfragen in elexiko gesammelt wurden, haben gezeigt, dass auch unerwartete Funde zutage treten, die außerhalb der eigenen linguistischen Kompetenz und Erwartbarkeit liegen und daher mit anderen Methoden möglicherweise nicht erfasst worden wären.

Deutlich schlechtere Ergebnisse erzielt die Suche nach Kollokatoren zur Extrahierung potentieller Kandidaten bei hochpolysemen Ausdrücken wie frei, bei denen einige wenige Lesarten stark im Korpus dominieren zu Ungunsten zahlreicher wenig auffälliger Lesarten. Ebenso schwierig ist diese Herangehensweise auch für Verben, die generell dazu tendieren, valenzartige Subjekt- oder Objektstrukturen in unmittelbarer Umgebung einzugehen, die mit Substantiven oder auch mit Adjektiven kollokieren und weniger systemhafte Konstruktionen mit Verben des gleichen Paradigmas aufweisen. Und deutlich eingeschränkter ist auch die Suche nach Synonymen, die teilweise in enger semantischer Nachbarschaft, aber tendenziell in größerer kontextueller Umgebung miteinander vorkommen. ${ }^{5}$ So zeigt etwa eine Kollokationsanalyse des Verbs akzeptieren zahlreiche erwartbare Substantive wie Entschuldigung, Friedensplan, Verhandlungspartner oder Spielregeln und auch adverbial

$5 \mathrm{Zu}$ einigen typischen Synonymkonstruktionen in unmittelbarem Kontext siehe Storjohann (2010). 
gebauchte Adjektive wie klaglos, stillschweigend, widerspruchslos, widerwillig, vorbehaltlos etc. aber kaum Verben, die eine Bedeutungsäquivalenz oder einen semantischen Gegensatz bezeichnen (siehe Abbildung 3).

\begin{tabular}{|c|c|c|c|c|c|}
\hline \multicolumn{6}{|c|}{ Ansicht Andocken Extras Hilfe } \\
\hline$\#$ & LLR & kumul. & Häufig & Kookkurrenzen & syntagmatische Kuster \\
\hline 9 & 744 & 3966 & 196 & klaglos & 458 klaglos $[\ldots]$ akzeptiert \\
\hline 10 & 738 & 4192 & 226 & stillschweigend & 528 stillschweigend [...] akzeptiert \\
\hline 11 & 509 & 4472 & 280 & allseits & $38 \%$ allseits $[\ldots]$ akzeptierten \\
\hline 12 & 481 & 4897 & 425 & Entschuldigung & $38 \%$ die Entschuldigung $[\ldots]$ akzeptiert \\
\hline 13 & 432 & 5039 & 142 & Murren & $50 z$ ohne Murren $[\ldots]$ akzeptiert \\
\hline 14 & 417 & 5147 & 108 & widerspruchslos & $53 \frac{2}{6}$ iderspruchslos akzeptiert \\
\hline 15 & 380 & 5302 & 155 & widerwillig & 448 nur widerwillig $[\ldots]$ akzeptiert \\
\hline 16 & 318 & 5388 & 86 & Schlichterspruch & 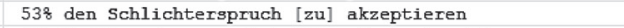 \\
\hline 17 & 299 & 5504 & 116 & vorbehaltlos & 48\% Rechtsordnung vorbehaltlos [zu] akzeptieren \\
\hline 18 & 296 & 5584 & 80 & Dr & 288 nicht|zu akzeptieren $[$ so $\ldots]$ Dr \\
\hline 19 & 293 & 5670 & 86 & Anderssein & 668 das Anderssein [... zu] akzeptieren und \\
\hline 20 & 261 & 5800 & 130 & Friedensplan & 338 den Friedensplan $[\ldots$ zu] akzeptieren \\
\hline 21 & 240 & 6001 & 201 & respektieren & $60 \mathrm{zu}$ akzeptieren [und $\mathrm{zu}$ ] respektieren \\
\hline 22 & 226 & 6128 & 127 & Verhandlungspartner & 508 als verhandlungspartner $[\ldots]$ akzeptiert ... werden \\
\hline 23 & 215 & 6155 & 27 & Toulas & 1008 und Ian Toulas griechische Großfamilie akzeptieren \\
\hline \multirow[t]{2}{*}{24} & 214 & 6156 & 1 & akzept humane & $100 \%$ akzeptierende $\ldots$ humane $\ldots$ akzept \\
\hline & & 6159 & 3 & akzept & 1008 akzept - für akzeptierende \\
\hline 25 & 197 & 6214 & 55 & Strafbefehle & 568 die Strafbefehle [nicht] akzeptiert \\
\hline 26 & 195 & 6286 & 72 & anstandslos & 548 anstandslos [...] akzeptiert \\
\hline 27 & 187 & 6474 & 188 & Spielregeln & 518 die Spielregeln [... zu] akzeptieren \\
\hline 28 & 170 & 6533 & 59 & Mehrheitsentscheidungen & 678 Mehrheitsentscheidungen [zu] akzeptieren \\
\hline 29 & 155 & 6582 & 49 & bzw & $28 \%$ bzw $[\ldots$ nicht $]$ akzeptiert werden \\
\hline 30 & 141 & 6625 & 43 & Mehrheitsentscheidung & 418 eineldie Mehrheitsentscheidung [zu] akzeptieren \\
\hline 31 & 140 & 6655 & 30 & EG-Karten & 66\% Euro EC-Karten [werden] akzeptiert \\
\hline 32 & 135 & 6786 & 131 & respektiert & $57 \%$ akzeptiert [und] respektiert werden \\
\hline 33 & 131 & 6834 & 48 & Schiedsspruch & 478 den Schiedsspruch $[\ldots$ zu] akzeptieren \\
\hline 34 & 128 & 6854 & 20 & Mehrheitsentscheide & 608 die ... Mehrheitsentscheide [zu/nicht] akzeptieren \\
\hline 35 & 127 & 8200 & 1346 & Urteil & 338 das Urteil [... nicht|zu] akzeptieren \\
\hline 36 & 127 & 8246 & 46 & kritiklos & 568 kritiklos $[\ldots]$ akzeptiert werden \\
\hline
\end{tabular}

Abbildung 3: Auszug aus der Kollokationsanalyse des Verbs akzeptieren

In solchen Fällen nutzt elexiko ein korpusgesteuertes Verfahren, bei dem Kollokationsprofile zwischen Ausdrücken miteinander kontrastiert werden, um semantisch ähnliche Ausdrücke zu einem Suchwort zu ermitteln (cf. Belica 2011). In elexiko wird dafür die Kookkurrenzdatenbank $\mathrm{CCDB}^{6}$ (Belica 2001ff.) mit der entsprechenden Funktionalität „related collocation profiles“ eingesetzt. Auf diese Weise werden u.a. für verbale Stichwörter Verben ermittelt, die über ein ähnliches Kollokationsprofil verfügen und potentielle Kandidaten für eine Synonymie- oder Gegensatzrelation sind. Diese sind empirisch und statistisch abgesichert, erhalten aber zunächst in elexiko nur den Status eines Kandidaten, den es zu prüfen gilt. Für das Suchwort akzeptieren sind es u.a.: respektieren, zustimmen, anerkennen, hinnehmen, abfinden, ablehnen, beugen, abrücken, annehmen, ignorieren, widersetzen, tolerieren, zulassen, opponieren, aufzwingen, unterstützen, zurückweisen (siehe Abbildung 4).

6 Kookkurrenzdatenbank CCDB siehe http://corpora.ids-mannheim.de/ccdb/. 


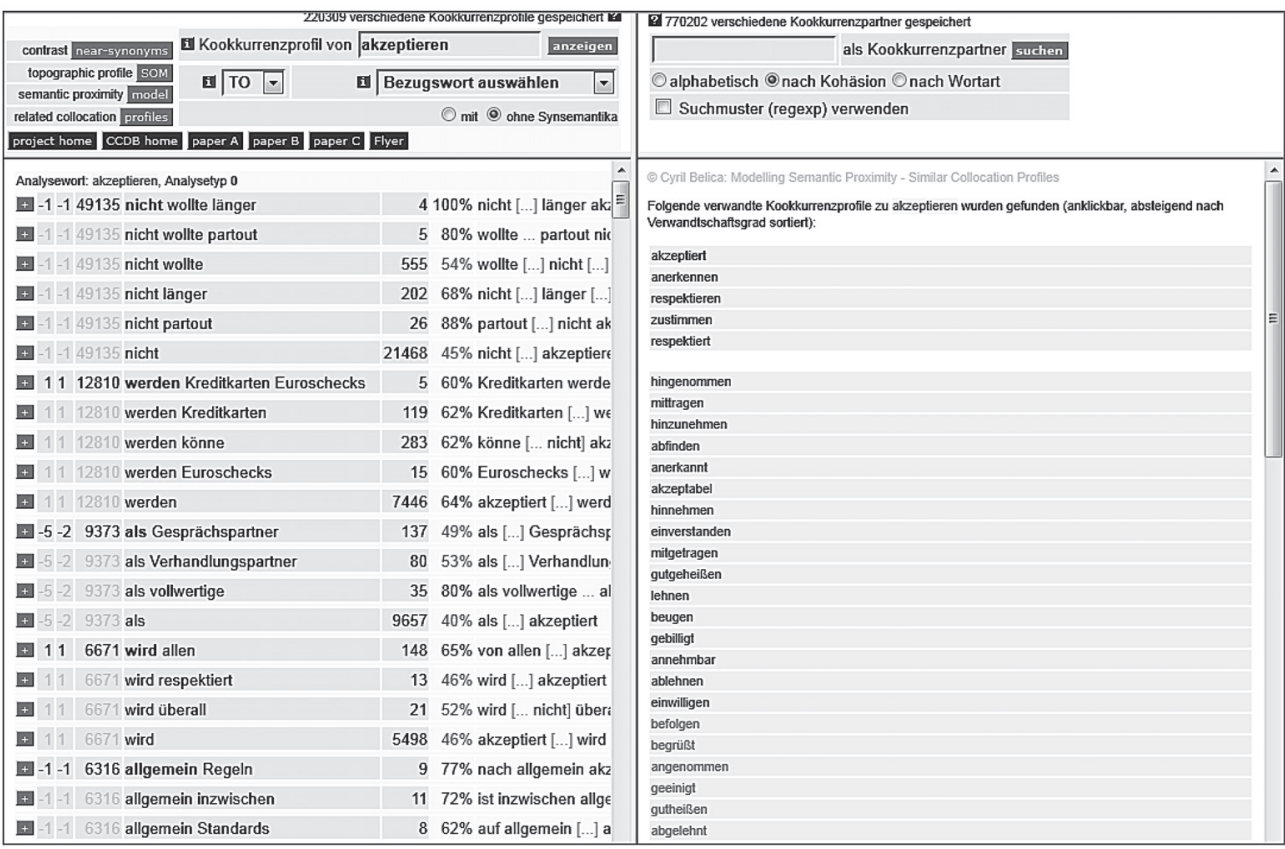

Abbildung 4: Ausschnitt aus der CCDB, verbale Ausdrücke mit ähnlichem Kollokationsprofil wie akzeptieren

Die angebotene Liste ist nicht das Ergebnis lexikografischer Interpretation, sondern ausschließlich das Ergebnis softwaregesteuerter Analysen. Daher ist ihr endgültiger Status zunächst ungeklärt. Je nach Ziel des Nachschlagewerkes wird unterschiedlich mit solchen Daten umgegangen. In elexiko werden die Ergebnisse nicht einfach automatisiert übernommen. Hier wird der Übergang zum korpusbasierten Vorgehen unerlässlich, um verlässliche Aussagen darüber treffen zu können, ob es sich um relevante Synonyme/Antonyme handelt. Nur über anschließende, gezielte datenbasierte Prüfanfragen kann u.a. geklärt werden, ob die automatisch vorgeschlagenen Partnerwörter hinreichend oft kontextuell belegt sind oder periphere Erscheinungen sind (siehe dazu Abschnitt 2.3).

Dagegen werden z.B. im Wortschatzportal der Universität Leipzig ${ }^{7}$ rein statistisch ermittelte, mögliche Relationspartner in alphabetisch geordneten Listen angeboten. In Abbildung 5 sind die Relationen des Ausdrucks frei, die automatisiert gewonnen werden, illustriert.

Die hier angegeben Korpusdaten enthüllen eine beeindruckende Vielfalt an möglichen Synonymen. Eine Analyse bezüglich der Symmetrie der angegebenen Synonyme zeigt ebenso, dass unter jedem aufgelisteten Synonym von achtbar bis zwanglos auch das Partnerwort frei als Rückverweis vorzufinden ist. Aber über eine quantitative korpusgesteuerte Analyse gehen diese Ergebnisse nicht hinaus. Die Synonyme erscheinen unkommentiert und sind kontextlos, und ohne semantische Zuordnung, wie sie ein stark polysemes Adjektiv wie frei

7 Wortschatzportal Universität Leipzig siehe: http://wortschatz.uni-leipzig.de/. 


\begin{abstract}
Relationen zu anderen Wörtern:
Synonyme: achtbar, allein, alleinstehend, astrein, autark, autonom, befreit, bieder, brav, disponibel, durchgängig, ehrbar, ehrenhaft, emanzipiert, entlassen, erlöst, formlos, fortschrittlich, frankiert, freigemacht, gebührenfrei, gelöst, gratis, großzügig, hemdsärmelig, improvisiert, kostenlos, lässig, ledig, leer, leger, modern, nachlässig, natürlich, natürlich, neuartig, offen, offen, progressiv, rein, salopp, selbständig, selbstverantwortlich, sinngemäß, souverän, supermodern, umsonst, unabhängig, unbebaut, unbefangen, unbehindert, unbelastet, unbeschränkt, unbeschwert, unbesetzt, uneingeschränkt, unentgeltlich, ungebunden, ungeniert, ungenutzt, ungezwungen, unkontrolliert, unkonventionell, unverdächtig, unverheiratet, unvorbereitet, unzeremoniell, vakant, verfügbar, zwanglos
\end{abstract}

vergleiche: emanzipiert, frank, gratis, vakant

ist Synonym von: abgespannt, ausgestoßen, ausgezogen, auswendig, autark, autonom, bloß, ehelos, eigenständig, emanzipiert, entblößt, entfesselt, enthemmt, enthüllt, enthüllt, entkleidet, entlastet, entlaubt, entspannt, familiär, gebührenfrei, geflohen, geschminkt, gratis, hemdsärmlig, hemmungslos, herrenlos, hüllenlos, improvisiert, informell, kahl, kostenfrei, kostenlos, kostenlos, lässig, ledig, leer, leger, licht, locker, los, lose, nackt, natürlich, nonchalant, offen, offen, offen, offenbar, offenherzig, salopp, selbständig, single, Stegreif, unabhängig, unbeaufsichtigt, unbefangen, unbehindert, unbekleidet, unbelastet, unbeobachtet, unbeschränkt, unbesetzt, unbestraft, unentgeltlich, ungebunden, ungehemmt, ungeniert, ungezwungen, unkontrolliert, unumwunden, unverheiratet, unverkrampft, unvorbereitet, unzeremoniell, vakant, verfügbar, vertraulich, vertraut, zügellos, zwanglos

wird referenziert von: gratis, ledig, offen, schrankenlos, unabhängig, ungebunden, vakant

Abbildung 5: Auszug aus dem Eintrag frei des Wortschatzportals

erfordern würde, ist eine derartige Auflistung für jeden Nichtmuttersprachler ,positively dangerous“ (Partington 1998, 47). Bei genauerer Betrachtung der dazugehörigen Stichworteinträge werden Details deutlich, die die Verlässlichkeit der angebotenen Informationen in Frage stellen. Ein Beispiel: Folgt man dem Eintrag zu supermodern ist dort das Synonym frei als einziges Synonym korrekt symmetrisch verzeichnet. Weiter im Eintrag gibt es Korpusbelege, die die Bedeutung des monosemen Ausdrucks supermodern, mit dem frei ja bedeutungsähnlich sein soll, veranschaulichen. In keinem der Belege ist aber frei als Synonym denkbar (cf. Abbildung 6).

Ausschließlich automatisiert extrahierte Daten maschineller Prozesse auf der Basis korpusgesteuerter Verfahren bergen das Risiko, fehlerhaft oder unzuverlässig zu sein. Dies steht in klarem Widerspruch zu den Ansprüchen der NutzerInnen an ein Nachschlagewerk. Laut einer Umfrage sehen NutzerInnen in der Zuverlässigkeit der Wörterbuchangaben eines der wichtigsten Kriterien (cf. Müller-Spitzer u.a. 2011). Eine korpusgesteuerte Ermittlung von sinnverwandten Ausdrücken nutzt auch elexiko, aber die Ergebnisse unterliegen der lexikografischen Analyse und Interpretation, einer Zuordnung zu entsprechenden Lesarten sowie einer kontextuellen Überprüfung eines jeden einzelnen Partnerwortes und der Bereitstellung eines entsprechenden Beleges, der jede dokumentierte Beziehung illustriert (detaillierter siehe Storjohann 2005). Die redaktionelle Arbeit in elexiko hat in der Vergangenheit gezeigt, dass, in Abhängigkeit des zu beschreibenden Stichwortes, die korpusgesteuerte Methode allein nicht der Qualitätssicherung genügen kann. Sie ist kein Garant dafür, alle relevanten Synonyme bzw. Antonyme im Korpus zu erfassen, um diese lexikografisch zu dokumentieren. Darüber hinaus stellt ein korpusgeleitetes Prozedere auch keine Gewährleistung dafür 


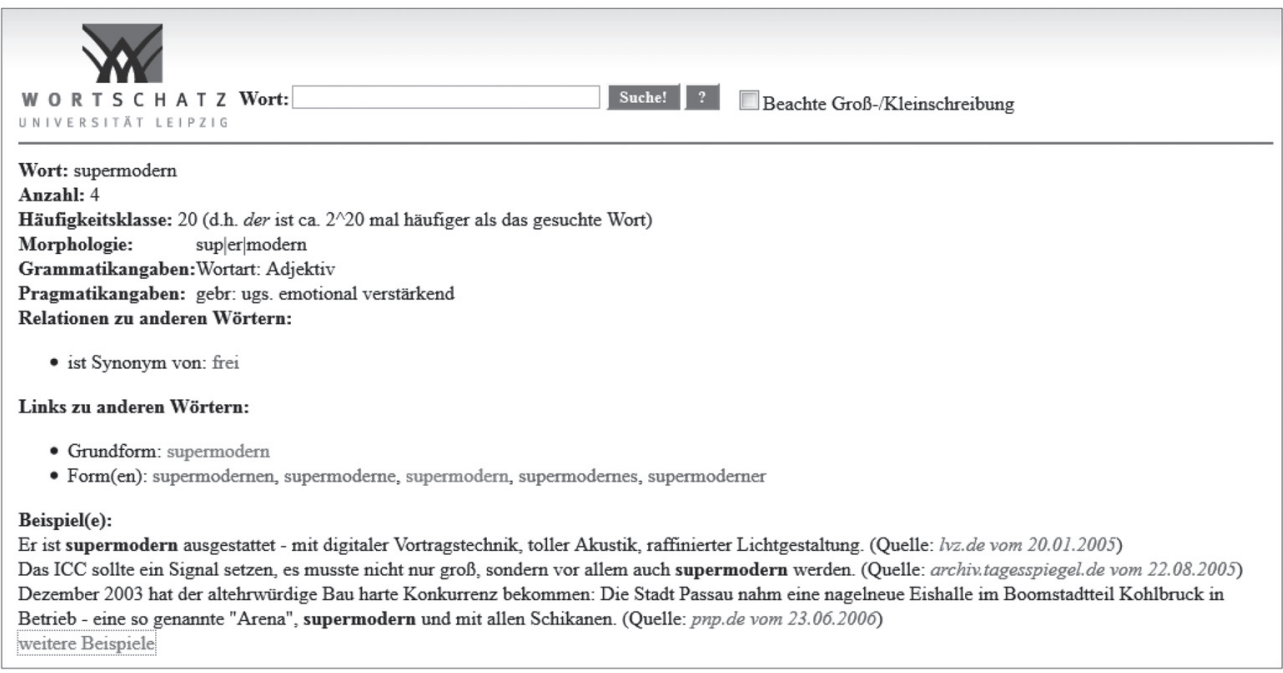

Abbildung 6: Eintrag supermodern mit Synonymangabe und Belegen

dar, Ausdrücke, die eine Antonymie- bzw. Synonymiebeziehung regelhaft eingehen, auch konsequent symmetrisch in einem Nachschlagewerk zu verzeichnen. Können diese beiden Mängel denn durch den Einsatz eines korpusbasierten Verfahrens im Sinne einer komplementären Methode behoben werden?

\subsection{Chancen und Probleme der korpusbasierten Ermittlung}

In einigen Fällen ist der ausschließliche Einsatz der korpusgeleiteten Methode nicht hinreichend nutzbar, um das semantische Beziehungsgeflecht, das ein Ausdruck zu seinen Bedeutungsäquivalenten und Gegensätzen eingeht, eruieren zu können. Bei der korpusbasierten Methode nutzen Lexikografinnen und Lexikografen das Korpus als quantitative Datenquelle und Prüfinstrumentarium, um lexikografische Annahmen zu einer bestehenden Sinnverwandtschaft zu bestätigen oder zu verwerfen, um nach Belegen für bestehende Hypothesen zu suchen oder um ein Phänomen quantitativ zu evaluieren. In solchen Fällen dienen introspektive Kompetenz und Angaben aus Sekundärquellen zunächst der Hypothesenbildung, die anhand der Korpusdaten gesucht, quantifiziert, interpretiert und verifiziert werden. In elexiko werden die Ergebnisse der datengeleiteten Kookkurrenzanalyse, die Resultate der Ermittlung von Ausdrücken mit ähnlichem Kollokationsprofil (siehe Abschnitt 2.2, Abbildung 4) sowie die Angaben weiterer Sekundärquellen gezielt kontextuell im Korpus überprüft. Das heißt, dass elexiko das korpusbasierte Verfahren als zusätzliche, nachgestellte und komplementäre Methode der Gewinnung von sinnverwandten Ausdrücken nutzt.

Korpusbasierte Untersuchungen stellen in manchen Fällen nur „Bonusmaterial“ (cf. Tognini-Bonelli 2001, 66) dar, in anderen Fällen sind sie die einzige Möglichkeit, umfangreiche und essenzielle Angaben bezüglich sinnverwandter Ausdrücke machen zu können. Dies gilt vor allem für stark polyseme und hochfrequente Ausdrücke, für die die korpusgesteuerte Methode Ergebnisse hervorbringt, die nur einer oder wenigen Lesarten zugeord- 
Beziehung(en) der Bedeutungsgleichheit/-äquivalenz

(i) Synonym(e):

anerkennen
annehmen
billigen
dulden

dulden wird nur eingeschränkt synonymisch zu akzeptieren verwendet. Häufig setzt die Handlung, die mit dulden bezeichnet wird, einen geringeren Grad an

Zustimmung des Handlungsträgers voraus, als es bei einer Handlung, die mit akzeptieren bezeichnet wird, der Fall ist. (Vgl. dazu den folgenden Beleg.)

Wer in der einen Szene Opfer ist, kann in der nächsten schon Täter sein. So der bosnische Serbe, als Flüchtling in Belgrad mehr geduldet als akzeptiert. (Züricher Tagesanzeiger, 18.06.1999, S. 20, Bilder der Gewalt.)

hinnehmen

hinnehmen wird nur manchmal synonymisch zu akzeptieren verwendet. Häufig setzt die Handlung, die mit hinnehmen bezeichnet wird, einen geringeren Grad an Zustimmung des Handlungsträgers voraus, als es bei einer Handlung, die durch akzeptieren bezeichnet wird, der Fall ist. (Vgl. dazu den folgenden Beleg.)

Reformen und Veränderungen im Bildungswesen und in der Gesellschaft erfolgen heute sehr schnell. Das ist eine Tatsache, die man akzeptieren muss.

Akzeptieren heisst für mich aber nicht, es einfach resignierend hinzunehmen. (St. Galler Tagblatt, 25.03.2000, Mitsprache mit Mitverantwortung.)

zulassen

zustimmen

Beziehung(en) des Bedeutungsgegensatzes

(i) komplementäre(r) Partner:

ablehnen

kritisieren

verurteilen

verweigern

weigern

Abbildung 7: Ausschnitt der sinnverwandten Wörter zu akzeptieren in elexiko 
net werden können, weil bestimmte Verwendungsweisen im Korpus sprachlich dominieren. Dann ist eine gezielte Korpusprüfung unerlässlich, um auch für weniger signifikante Verwendungen Informationen zu Relationswörtern angeben zu können. Gerade Fremdsprachenlernende haben ein Interesse, im Wörterbuch Synonymangaben auch bei weniger geläufigen Gebräuchen zu finden. So wird beispielsweise ein potentielles Synonym $Y$ eines Suchwortes $X$, im Abstand von ca. 2 Sätzen im Korpus miteinander in beliebiger Reihenfolge gesucht. Die in Abbildung 4 dargestellten potentiellen synonymen Kandidaten zum Suchwort akzeptieren werden hinsichtlich ihrer Relevanz lexikografisch „,manuell“ überprüft. Eine korpusbasierte Abfrage ist in elexiko auch immer gleichzeitig die Suche nach geeigneten Belegen zur authentischen Illustrierung von sprachlichen Beziehungen. Bei der Überprüfung der gemeinsamen Textstellen wird z.B. deutlich, dass Ausdrücke wie dulden oder hinnehmen Verwendungsrestriktionen aufweisen. Alle gewonnenen Erkenntnisse werden daraufhin in der lexikografischen Beschreibung der Relationswörter u. a. in Form von Belegen und Kommentaren integriert (siehe Abbildung 7).

Aber neben dem Prüfen automatisch extrahierter Kandidaten und den zusätzlichen Erkenntnissen zu synonymen Einschränkungen wird die korpusbasierte Methode genutzt, um mögliche, vorwiegend weniger signifikante Beziehungen auch quantitativ zu erfassen und auszuwerten. Das hochpolyseme Stichwort frei (siehe Wörterbuchartikel unter: http:// www.owid.de/artikel/272789), mit seinen 15 unterschiedlichen kontextuellen Verwendungen kann nicht allein auf der Basis eines korpusgesteuerten Verfahrens disambiguiert werden, und entsprechend bietet auch die statistische Auswertung unmittelbarer Kontextwörter nicht hinreichend Informationen zum sinnrelationalen Geflecht sämtlicher Lesarten und ihrer semantischer Nuancierungen. So konnten z.B. die Synonyme nackt, unbegrenzt, ungeregelt nicht durch automatische Analysen generiert werden. Sie sind z.B. auch nicht im entsprechenden Eintrag des Wortschatzportals aufgelistet. Die korpusbasierte Recherche nach diesen Ausdrücken zeigt aber, dass diese Ausdrücke mehrfach in bedeutungsähnlichen Kontexten belegt sind, als relevant eingestuft werden können und damit lexikografisch kontextuell eingeordnet, dokumentiert und belegt werden können. In elexiko sind sie daher für das Stichwort als Synonyme mit folgenden Belegen gebucht:

nackt - eingeordnet in der Lesart: ,nicht umgeben oder unbedeckt', Spezifizierung: ,nackt

1. Wenn in den Sportumkleidekabinen die High-School-Jungs nicht nur freien Oberkörper, sondern auch nackten Hintern zeigen, kreischen die Mädels im Zuschauersaal laut auf, als wär man bei den „Chippendales“. (die tageszeitung, 12.11.1998, S. 29)

unbegrenzt - eingeordnet in der Lesart: ,unbehindert`

2. Für Gray sind die neuen Erlösungsfantasien von erschreckender Simplizität: Der unbegrenzte Weltmarkt als Heimstatt der aufgeklärten Vernunft, der Homo oeconomicus als Vollender der Moderne. Irrig ist dies laut Gray vor allem deshalb, weil der freie Markt alles andere als eine historische Notwendigkeit sei. (Frankfurter Rundschau, 29.10.1999, S. 7)

ungeregelt - eingeordnet in der Lesart: ,unbehindert':

3. Der Beinahe-Zusammenbruch des Dualen Systems zeigt, wie berechtigt das Mißtrauen in die Kräfte des freien Marktes bei Umweltfragen ist. In einer absolut ungeregelten Marktwirtschaft würden die Schwachen untergehen, zu ihrem Schutz haben wir die soziale Marktwirtschaft. (Frankfurter Allgemeine Zeitung, 00.00.1993) 
Die Synonym- bzw. Antonymangaben sind also nicht zentral für ein Stichwort erfasst, sondern sie werden kontextabhängig dokumentiert. Ohne korpusbasierte Suchanfragen hätten diese Synonyme nicht in elexiko erfasst werden können.

In der lexikografischen Praxis von elexiko hat sich der kombinierte Einsatz unterschiedlicher Strategien (multi-method approach) und die konsequente Dateninterpretation als sehr gewinnbringend erwiesen. Die lexikografisch angelegte Disambiguierung und die Zuordnung synonymer/antonymer Ausdrücke in die entsprechenden Lesarten stellen einen erheblichen Mehrwert gegenüber rein automatisierten Listen dar. Die computergesteuerte korpusgeleitete Gewinnung von Synonymen oder Antonymen bringt statistisch signifikante Regularitäten systematisch zum Vorschein. Die korpusgesteuerte Ermittlung von Ausdrücken mit ähnlichem Kollokationsprofil stellt einen zusätzlichen Gewinn für solche Suchwörter dar, für die die statistische Kookkurrenzanalyse wenig bis gar keine paradigmatischen Partnerwörter extrahieren kann. Aber nur die lexikografische Überprüfung der Daten ermöglicht Einblicke in Verwendungsbeschränkungen, gewährleistet die entsprechende kontextuelle Einordnung und bietet somit insgesamt eine deutlich höhere Authentizität und Zuverlässigkeit der Wörterbuchangaben. Die dokumentierten Wörterbuchinformationen sind empirisch fundiert, inhaltlich bestätigt, quantitativ evaluiert und lexikografisch kontextuell eingeordnet. Aber gewährleistet diese Strategie nun Wörterbucheinträge mit einer stärkeren Konsistenz von bilateralen Beziehungen und mit konsequent symmetrischen Verweisen?

\section{Methoden konsistenter sinnrelationaler Vernetzungsstrukturen}

Im Jahr 2010 wurde in elexiko eine Auswertung vorgenommen, bei der der damalige Stichwortbestand von ca. 1400 Stichwörtern mit all ihren Lesarten und ihren sinnverwandten Wörtern, die mit den oben erwähnten Methoden gewonnen wurden, dahingehend überprüft wurde, ob alle ein- und ausgehenden Relationen in beiden Richtungen korrekt eingetragen sind. ${ }^{8}$ Das Ergebnis war, dass 2558 bidirektionale Vernetzungen (1279 Wortpaare) korrekt auf Lesartenebene angegeben wurden. 1700 Vernetzungen waren dagegen nicht symmetrisch miteinander vernetzt. Die aufgedeckte Lückenhaftigkeit ist auf mehrere Ursachen zurückführen. Zum einen passierte es, dass ein Partnerwort schlichtweg während des lexikografischen Prozesses vergessen wurde. Außerdem kam es vor, dass auf eine falsche Lesart, also auf einen falschen Kontext bei polysemen Ausdrücken verlinkt wurde. Die Mehrzahl der Fehler aber unterliefen, weil zahlreiche Stichwörter $A, B, C$ etc. zum Zeitpunkt der Erarbeitung eines Stichwortes $X$ nicht redaktionell ausgearbeitet sind, weil elexiko kein abgeschlossenes Projekt ist. Zum Zeitpunkt der Bearbeitung von $X$ kann daher nicht auf $A, B$ oder $C$ verlinkt werden, weil diese Stichwörter noch nicht über ein ausgearbeitetes Bedeutungsspektrum verfügen. Wurde eines der bereits als Beziehungspartner dokumentierten Stichwörter $A, B$ oder $C$ später ausgearbeitet, wurde vermutlich vergessen, die für einen korrekten Verweis zusätzlich benötigten Informationen, wie Angabe der entsprechenden Lesart, im Stichwort $X$ nachzutragen.

8 Derzeit sind ca. 1700 Stichwörter in elexiko vollständig redaktionell ausgearbeitet. 
Das Ergebnis der Auswertung zeigt deutlich, dass nicht ein bestimmtes Prozedere oder die Kombination verschiedener Korpusverfahren das Instrument der Konsistenzsicherung sind. Selbst wenn sämtliche relevante Beziehungen auf die eine oder andere Art ermittelt werden, ist das kein Garant dafür, dass diese auch konsequent symmetrisch dokumentiert werden, auch dann nicht, wenn das Wörterbuch inhaltlich abgeschlossen ist und alle Stichwörter bearbeitet sind. Hält man sich noch einmal die Vielzahl an sinnrelationalen Beziehungen für ein hochpolysemes und hochfrequentes Stichwort wie frei vor Augen (siehe Abbildung 8) ${ }^{9}$, wird deutlich, dass diese Geflechte nicht intuitiv systematisch abrufbar sind.

Die Komplexität des Beziehungsnetzes für den Ausdruck frei illustriert die folgende Abbildung, welche Beziehungen zusammengehöriger Kontexte aufgliedert.

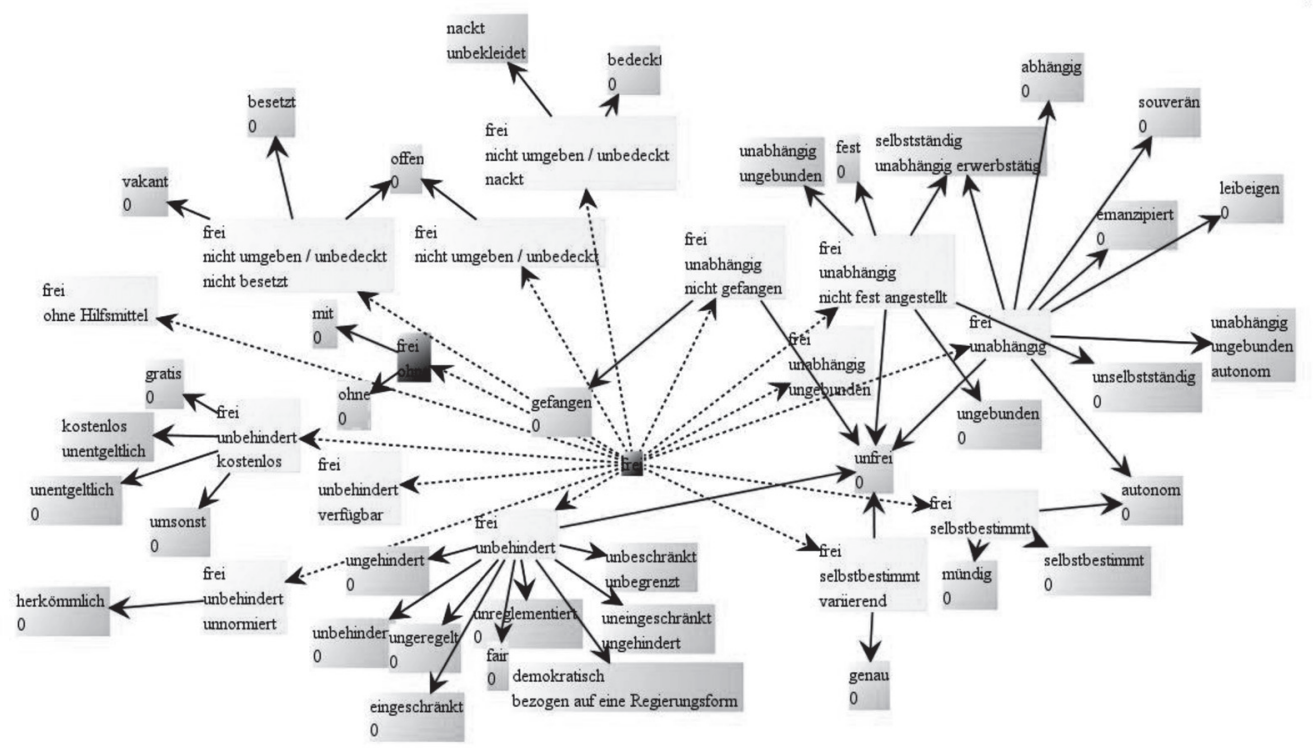

Abbildung 8: Ausgehende lesartengebundene synonyme Vernetzungen des Ausdrucks frei

Die größten Bemühungen auf redaktioneller Seite scheitern beim Versuch, alle Vernetzungen introspektiv korrekt abzurufen und daraufhin symmetrisch anzulegen. Müller-Spitzer (2010, 150f.) weist darauf hin, dass es sich schlichtweg um menschliches Versagen handelt. Alle sinnverwandten Ausdrücke sind, sofern sie selbst Stichwort des Wörterbuches sind, als lesartenbezogener Link angelegt, um eine schnelle Navigation zu diesen Stichwörtern in

9 Die Abbildung hat Dr. Peter Meyer, wissenschaftlicher Mitarbeiter des IDS, erstellt. Die Rechtecke enthalten den Relationspartner (oben) und darunter die entsprechende Lesart und, falls vorhanden, die relevante semantische Spezifizierung, für die eine Beziehung besteht. Bei der mit Schrägstrich markierten Bezeichnung einer Lesart handelt es sich um eine Doppelbenennung Die in der Grafik mit „, “ markierten Partnerwörter verfügen noch nicht über ein ausgearbeitetes Lesartenspektrum. Die Beziehung wird daher zunächst auf Stichwortebene und nicht auf Kontextebene (Lesartenebene) angesetzt. 
ihren jeweils relevanten sinnrelationalen Verwendungen zu gewährleisten. Die Verlinkungen sind also nicht auf der Ebene der Stichwörter angelegt, sondern auf Lesartenebene, um spezifische Beziehungen rein kontextuell zu erfassen. Damit gelangt man von einem Stichwort in einer bestimmten Verwendung zu einem seiner Synonyme oder Gegensatzwörter in den entsprechenden Kontexten und erfasst, in welchen konkreten Verwendungssituationen beispielsweise eine bedeutungsähnliche oder oppositionelle Beziehung vorliegt. Anders als bei rein lemmabasierten Zuordnungen (z.B. bei Wörterbucheinträgen im Leipziger Wortschatzportal), liegt bei elexiko die Herausforderung darin, dass bei der Erarbeitung eines Wörterbuchartikels Informationen zu den entsprechenden Verlinkungen sehr detailliert und lesartengebunden in der Artikelstruktur hinterlegt werden müssen.

Diese Art der Vernetzung ist nicht ohne Computerunterstützung zu leisten. Daher sollte für die Überprüfung von Wörterbuchvernetzungen eine Management-Software zum Einsatz kommen, die Beziehungen zu verschiedenen Stichwortebenen und zwischen verschiedenen Beziehungsarten prüfen kann (cf. Müller-Spitzer 2010). Für ein elektronisches Wörterbuch müssen dafür bestimmte technische Voraussetzungen geschaffen werden.

Vernetzungen werden in den elexiko-Artikeln technisch durch XML-Elemente im verweisenden Artikel repräsentiert; das Verweisziel wird darin durch eindeutige Kennungen (Zahlen bzw. Wörter oder Wortfolgen) spezifiziert, die dem Artikel als ganzen sowie auch z.B. seinen einzelnen Lesarten über XML-Attribute zugeordnet sind (cf. auch Müller-Spitzer 2010). Um die Bidirektionalität und Konsistenz etwa einer Antonymie-Vernetzung zu prüfen, müssen die zugehörigen XML-Elemente in den beiden Artikeln aufgesucht und die Vernetzungsinformationen miteinander verglichen werden. (Storjohann/Meyer 2012, 130)

Zur Sicherstellung von sinnrelationalen Beziehungen wurden in elexiko alle dafür notwendigen technischen Gegebenheiten der Redaktionsumgebung hergestellt, wie z.B. eine Schnittstelle zum XML-Editor, Zugriff auf die Datenbank, in der die Artikelinstanzen hinterlegt sind, etc. (siehe Meyer 2010, Klosa 2011b: 14-16, Müller-Spitzer 2010, MüllerSpitzer/Schneider 2009). Im Rahmen des Projektes BZVelexiko ${ }^{10}$ konnte dann ein solches Tool, der Vernetzungsmanager vernetziko, entwickelt werden, der seit 2010 bei der täglichen Artikelarbeit eingesetzt wird. Abbildung 9 demonstriert am Beispiel des Artikels frei die Benutzeroberfläche des Vernetzungsmanagers. Im oberen Bereich befinden sich diejenigen Stichwörter, die eine Beziehung auf den Artikel frei dokumentiert haben. Zusätzlich werden alle Informationen angegeben, die an diese Beziehung gebunden sind, wie die relevante Lesart oder eine relevante semantische Spezifizierung. Im unteren Bereich werden alle ausgehenden Beziehungen bzw. Beziehungsarten von frei für die entsprechenden Lesarten aufgeführt, die zum Zeitpunkt der Erarbeitung dokumentiert wurden. Der Vernetzungsmanager erteilt Auskunft über den Status der Beziehung (siehe Spalte „Vernetzungsstatus“), also darüber, ob eine Zuweisung der entsprechenden Lesart eines Stichwortes nicht erfolgt oder etwa, ob bei einem synonymen Ausdruck der Verweis in die Gegenrichtung fehlt. Informationen zum jeweiligen Vernetzungsstatus werden nach Auswählen einer Beziehung unterhalb der Tabelle zusätzlich kurz erläutert.

10 Zum Projekt BZVelexiko (Benutzeradaptive Zugänge und Vernetzungen in elexiko) siehe http:// www.ids-mannheim.de/lexik/BZVelexiko/. Der Vernetzungsmanager wurde in Zusammenarbeit mit Dr. Peter Meyer entwickelt. 


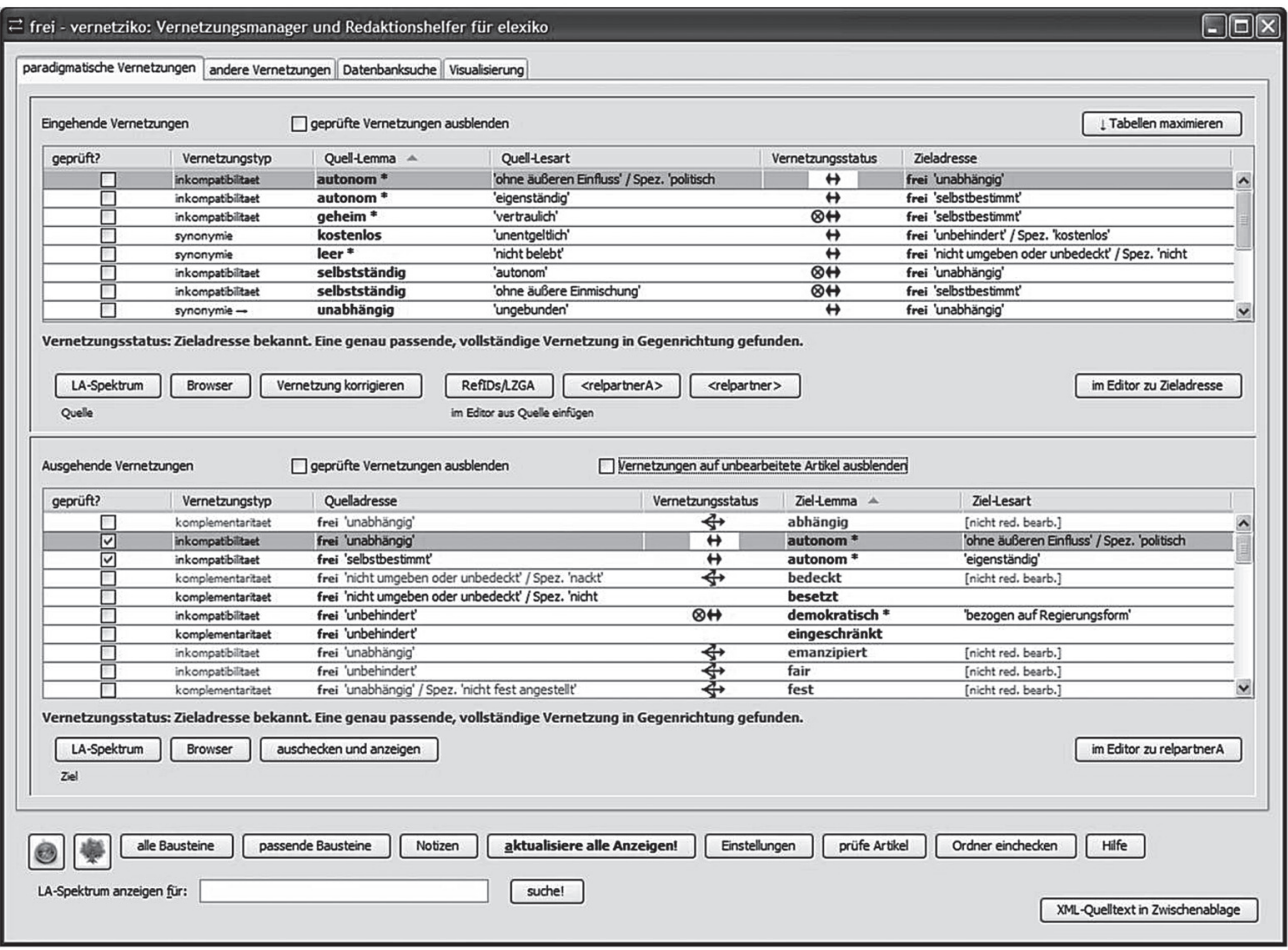

Abbildung 9: Vernetzungsmanager für elexiko-Wörterbuchartikel frei bei Korrekturarbeiten

Elexiko hat seitdem zahlreiche Fehler beseitigen, relevante Angaben, die zur Vernetzungsinformation gehören, nachtragen oder Partnerwörter ergänzen können. Gegenwärtig ist das Tool integrativer Bestandteil der redaktionellen Artikelarbeit, um bereits während des lexikografischen Prozesses alle nötigen Schritte zur Konsistenzherstellung durchführen zu können.

\section{Schlussbemerkungen}

In elexiko unterliegt die Auswahl der sinnverwandten Wörter primär Kriterien, die an den sprachlichen Usus gebunden sind und im Korpus eine ermittelbare Signifikanz aufweisen. Ziel von elexiko ist es, neben einem breiten Spektrum an Angaben vor allem authentische und zuverlässige Informationen für Nachschlagende kontextgebunden bereitzustellen. Dafür erweist sich das Zusammenspiel aus korpusgesteuertem und korpusbasiertem Ansatz als besonders geeignet, um optimale Erkenntnisse über verschiedene paradigmatische Konstruktionen zu erhalten, wie sie auch im tatsächlichen Sprachgebrauch realisiert werden. Bei der täglichen lexikografischen Arbeit mit einem Korpus und dem gewählten methodologischen Vorgehen werden die Diskrepanzen zur eigenen Intuition, zu stärker traditionell ori- 
entierten Synonymiken oder zu ausschließlich automatisch extrahierten Relationsangaben sichtbar. Intuitiv Erwartbares wird manchmal bestätigt, manchmal widerlegt, und Unerwartetes wird zum Teil als reguläres Phänomen evident. Beides wird mit Korpusdaten systematisierbar und gezielt überprüfbar gemacht. Diese "unexpectedness of the findings" (Tognini-Bonelli 2001, 48) stellt einen großen Gewinn bei der Arbeit mit einem umfangreichen Korpus in elexiko dar. Aber die Korpusbasis, die entsprechenden Analyse- und RetrievalTools und die gewählten Ansätze garantieren nicht, wie Paradis/Willners (2006) vermuten, „,a symmetry of presentation“. Für ein Printwörterbuch oder für ein elektronisches Nachlagewerk gilt, dass konsistente Verweis- oder Vernetzungsstrukturen nur mit zusätzlicher Softwareunterstützung gewährleistet werden können, denn nur diese kann Fehler oder inhaltliche Lücken ermitteln und korrigieren, für die man aus metalexikografischer Sicht nach vermeintlich nachvollziehbaren und transparenten Prinzipien sucht. Die Gewinnung von Synonymen oder Antonymen kann aus linguistischer Sicht durchaus sehr strikten linguistischen oder methodologischen Kriterien unterliegen, die sich jedoch nicht immer in der lexikografischen Präsentation nachvollziehen lassen.

Der lexikografische Aufwand zur Gewinnung, Analyse und Dokumentation von sinnrelationalen Ausdrücken ist trotz leistungsstarker Computerwerkzeuge in elexiko hoch. Der Einsatz von Recherchewerkzeugen und die Entwicklung maßgeschneiderter Tools haben die lexikografische Praxis in elexiko positiv verändert und die Wörterbuchbeschreibungen objektiver und inhaltlich konsistenter gemacht. Die Weiterentwicklung solcher oder ähnlicher Software wird auch in Zukunft die Arbeit der Lexikografinnen und Lexikografen verändern. Besonders in der englischen Lexikografie werden bereits Werkzeuge genutzt, die Daten inhaltlich nach diversen Merkmalen stärker ,stromlinienförmig ' bündeln. ${ }^{11}$ Die computationellen Prozesse der so genannten „Streamline-Tickboxlexikografie“ vermögen es, sprachliche Daten hinsichtlich unterschiedlicher linguistischer Kriterien automatisiert zu extrahieren, inhaltlich zu bündeln und stark vorsystematisiert anzubieten. Aufgrund ihrer fortlaufenden „Lernfähigkeit“ vermögen sie es außerdem, die kompetenzgesteuerte Selektion der gewonnenen Daten seitens der LexikografInnen nachzuvollziehen und abzuspeichern, um bei weiteren Anfragen die Suchergebnisse weiter nach erlernten Wünschen zu optimieren. Die Daten können dann automatisiert, ohne weiteres Eingreifen des Lexikografen/der Lexikografin in das entsprechende Dokument an die ausgewiesenen Stellen überführt werden. Diese Methode wurde z.B. im Projekt Dante - A lexical database for English erprobt. Neue Prozesse mit automatischer Datenauswahl werden die Wörterbucharbeit, und damit wohl auch die Methoden zur Ermittlung von sinnverwandten Ausdrücken, künftig weiter verändern. Neue lexikografische Modelle werden bereits entwickelt (cf. Rundell/ Kilgarriff 2011).

In this model, we envisage a change from the current situation, where the corpus software (some version of the word sketches) presents data to the lexicographer in (as we have seen) intelligently pre-digested form, to a new paradigm where the software selects what it believes to be relevant data and actually populates the appropriate fields in the dictionary database. In this way of working, the lexicographer's task changes from selecting and copying data from the software, to validating - in the dictionary writing system - the choices made by the computer. Having deleted or adjusted

11 Einen derartigen computergesteuerten Prozess bietet z.B. das Tool Sketch Engine (http://www. sketchengine.co.uk/) an, ein Korpusanalysesystem, das die Verwendungsmöglichkeiten eines Ausdrucks nach grammatischen und kollokationalen Kriterien zusammenfasst. 
anything unwanted, the lexicographer then tidies up and completes the entry. (Rundell/Kilgarriff 2011, 278)

Die gegenwärtige und wohl auch künftige Wörterbucharbeit ist ohne große elektronische Korpora, ohne Computertechnologien und ohne komplexe Automatisierungsprozesse undenkbar. Sie sollte aber die lexikografische Dateninterpretation nicht ausschließen müssen. Ob es künftig überhaupt noch LexikografInnen geben wird (cf. Grefenstette 1998, Rundell 2002, Rundell/Kilgarriff 2011), hängt zum einen davon ab, wie zuverlässig und authentisch NutzerInnen ihre Wörterbücher haben möchten, aber zum anderen auch davon, wie gut es LexikografInnen gelingen wird, ihre veränderte Rolle bei der Entstehung von Wörterbüchern vorteilhaft wahrzunehmen.

\section{Literatur}

\section{$5.1 \quad$ Wörterbücher}

Collins COBUILD 2003 = Sinclair, John (ed.): English Advanced Learner's Dictionary. 4. Auflage. Glasgow: Harper Collins Publishers, 2003.

elexiko = elexiko. http://www.owid.de/wb/elexiko/start.html

Wortschatzportal $=$ Wortschatzlexikon. Wortschatzportal der Universität Leipzig. $<$ http://wortschatz. uni-leipzig.de/>

\section{$5.2 \quad$ Literatur}

Belica 1995 = Belica, Cyril: Statistische Kollokationsanalyse und Clustering. Korpusanalysemodul. Mannheim: Institut für Deutsche Sprache, 1995.

Belica 2001ff. = Belica, Cyril: Kookkurrenzdatenbank CCDB - V3.2. Eine korpuslinguistische Denk- und Experimentierplattform für die Erforschung und theoretische Begründung von systemisch-strukturellen Eigenschaften von Kohäsionsrelationen zwischen den Konstituenten des Sprachgebrauchs. Mannheim: Institut für Deutsche Sprache, 2001ff. <http://corpora.ids-mannheim. $\mathrm{de} / \mathrm{ccdb} />$

Belica 2011 = Belica, Cyril: Semantische Nähe als Ähnlichkeit von Kookkurrenzprofilen. In: Abel, Andrea/Zanin, Renata (edd.): Korpora in Lehre und Forschung. Bozen-Bolzaro: Freie Universität, 2011, 155-178.

Cruse 1986 = Cruse, Alan: Lexical Semantics. Cambridge: Cambridge University Press, 1986.

Grefenstette 1998 = Grefenstette, Gregory: The Future of Linguistics and Lexicographers: Will there be Lexicographers in the year 3000? In: Fontenelle, Thierry et al. (edd.): Proceedings of the $8^{\text {th }}$ EURALEX International Congress on Lexicography in Liege, Belgium. Liege: University of Liege, English and Dutch Departments, 1998, 25-41.

Jones 2002 = Jones, Steven: Antonymy: a corpus-based perspective. London/New York: Routledge, 2002.

Jones et al. 2012 = Jones, Steven et al.: Antonyms in English: Construals, Constructions and Canonicity. Studies in English Language. Cambridge: Cambridge University Press, 2012.

Justeson/Katz 1991 = Justeson, John S./Katz, Slava M.: Redefining Antonymy: The Textual Structure of a Semantic Relation. In: Literary and Linguistic Computing 7, 1991, 176-184.

Meyer 2011 = Meyer, Peter: vernetziko: A Cross-Reference Management Tool for the Lexicographer's Workbench. In: Conference Proceedings of eLEX2011 (Electronic Lexicography in the 21st 
Century: New Applications for New Users), 10-12 November 2011, Bled, Slovenia. Bled, eLEX, 2011. <http://www.trojina.si/elex2011/Vsebine/proceedings/eLex2011-25.pdf>

Müller-Spitzer 2010 = Müller-Spitzer, Carolin: The Consistency of Sense-Related Items in Dictionaries: Current Status, Proposals for Modelling and Applications in Lexicographic Practice. In: Storjohann, Petra (ed.): Lexical-Semantic Relations. Theoretical and Practical Perspectives. Amsterdam: John Benjamins, 2010, 145-162 (Lingvisticæ Investigationes Supplementa 28).

Müller-Spitzer/Koplenig/Töpel 2011 = Müller-Spitzer, Carolin/Koplenig, Alexander/Töpel, Antje: What Makes a Good Online Dictionary? - Empirical Insights from an Interdisciplinary Research Project. In: Conference Proceedings of eLEX2011 (Electronic Lexicography in the $21^{\text {st }}$ Century: New Applications for New Users), Bled, Slovenia. Bled, eLEX, 2011. <www.trojina.si/elex2011/ Vsebine/proceedings/eLex2011-27.pdf>

Paradis/Willners 2006 = Paradis, Carita/Willners, Caroline: What a Corpus Based Dictionary Tells us about Antonymy. In: Corino, Elisa/Marello, Carla/Onesti, Christina (edd.): Proceedings of the 12th EURALEX International Congress 2006, Turin. Band 1. Turin: Edizioni dell'Orso Alessandria, 2006, 213-219.

Paradis/Willners 2007 = Paradis, Carita/Willners, Caroline: Antonyms in Dictionary Entries: Methodological Aspects. In: Linguistica 61/3, 2007, 261-277.

Rundell 2002 = Rundell, Michael: Good Old-Fashioned Lexicography: Human Judgment and the Limits of Automation. In: Corréard, Marie-Hélène (ed.): Lexicography and Natural Language Processing. A Festschrift in Honour of B. T. S. Atkins. Grenoble: EURALEX, 2002, 138-155.

Rundell/Kilgarriff 2011 = Rundell, Michael/Kilgarriff, Adam: Automating the Creation of Dictionaries: Where will it all End? In: Meunier, Fanny et al. (edd.): A Taste for Corpora. In Honour of Sylviane Granger. Amsterdam/Philadelphia: John Benjamins, 2011, 257-281.

Sinclair 1991 = Sinclair, John: Corpus, Concordance, Collocation. Oxford: Oxford University Press, 1991.

Sinclair 2002 = Sinclair, John: Intuition and annotation - the discussion continues. In: Aijmer, Karin/ Altenberg, Bengt (edd.): Advances in Corpus Linguistics. Papers from the 23rd International Conference on English Language Research on Computerized Corpora (ICAME 23). Göteborg 2002, 39-59.

Storjohann 2005 = Storjohann, Petra: Paradigmatische Relationen. In: Haß, Ulrike (ed.): Grundfragen der elektronischen Lexikographie. elexiko - das Online-Informationssystem zum deutschen Wortschatz. Berlin/New York: de Gruyter, 2005, 249-264.

Storjohann 2006 = Storjohann, Petra: New Lexicographic Approaches to the Description of Sense Relations. In: Corino, Elisa/Marello, Carla/Onesti, Christina (edd.): Proceedings of the 12th EURALEX International Congress 2006, Turin. Band 2. Turin: Edizioni dell'Orso Alessandria, 2006, 1201-1212.

Storjohann 2010 = Storjohann, Petra: Synonymy in Corpus Texts - Conceptualisation and Construction . In: Storjohann, Petra (Hg.): Lexical-Semantic Relations. Theoretical and Practical Perspectives. Amsterdam: John Benjamins, 2010, 69-94 (Lingvisticæ Investigationes Supplementa 28).

Storjohann/Meyer 2012 = Storjohann, Petra/Meyer, Peter: Konsistent vernetzte Wörterbucheinträge. In: Zeitschrift für Angewandte Linguistik 56, 2012, 121-134.

Tognini-Bonelli 2001 = Tognini-Bonelli, Elena: Corpus Linguistics at Work. New York/Amsterdam: John Benjamins, 2001.

\subsection{Elektronische Quellen}

BZVelexiko = BZVelexiko: Benutzeradaptive Zugänge und Vernetzungen in elexiko. <http://www1. ids-mannheim.de/lexik/BZVelexiko/>

CCDB $=$ Kookkurrenzdatenbank CCDB.$<$ http://corpora.ids-mannheim.de/ccdb/>

COSMAS II = Corpus Search, Management and Analysis System (COSMAS). <http://www.idsmannheim.de/cosmas2/uebersicht.html>

Dante $=$ Dante. A Lexical Database for English. <http://www.webdante.com/index.html $>$

Sketch Engine $=$ Sketch Engine. $<$ http://www.sketchengine.co.uk/?page=Website/SketchEngine $>$ 
